\title{
The Formation of G-band Bright Points I: Standard LTE Modelling
}

\author{
D. Kiselman
}

The Swedish Royal Academy of Sciences, Stockholm Observatory, SE-133 36 Saltsjöbaden, Sweden

\author{
R. J. Rutten \\ Sterrekundig Instituut, Postbus 80 000, NL-3508 TA Utrecht, The \\ Netherlands
}

B. Plez

GRAAL, Université de Montpellier II, FR-34095 Montpellier Cedex 5, France

\begin{abstract}
Assuming LTE, we investigate the formation of the $\mathrm{G}$ band in the semiempirical flux-tube model of Briand \& Solanki (1995). Preliminary results agree with observations of G-band bright-point contrast, even though the observed contrast depends sensitively on the amount of scattered light. It is the weakening of spectral lines in the flux tube that makes the bright-point contrast greater in the $\mathrm{G}$ band than in ordinary continuum. We also present LTE synthetic spectra for the full wavelength range from UV to IR. This serves to point out other promising pass bands for the observations of flux-tube structures.
\end{abstract}

\section{Spectrum synthesis of G-band bright points}

G-band filtergrams are often used for proxy magnetometry since photospheric bright points associated with magnetic structures show up so well in them (e.g. Berger et al. 1995). We have made LTE spectrum synthesis of the $G$ band (formed by $\mathrm{CH}$ ) in the semi-empirical magnetic flux-tube model NCHROM7 of Briand \& Solanki (1995) which is a refinement of the models of Bruls \& Solanki (1993) and Solanki (1986). This model is hereafter called "the flux tube" and it was used together with a surrounding model of quiet photosphere. The resulting intensity spectra were integrated using the transmission profiles of two filters that have been used at the Swedish Vacuum Solar Telescope on La Palma. $G$ for Gband and $C$ for the nearby "continuum band". These results were compared to a pair of $G$ and $C$ images of a solar active region (Berger \& Löfdahl, private communication). The images were acquired with the Swedish Vacuum Solar Telescope on La Palma and restored using phase-diversity techniques.

The hot bright walls of the (rotationally symmetric) flux tube create a bright ring seen in Fig. 1 as a double peak in the radiation temperature. Figure 1 also shows the temperature structure from three lines of sight of the model: quiet sun, the wall where the outgoing intensity is highest, and the centre. The contrast 


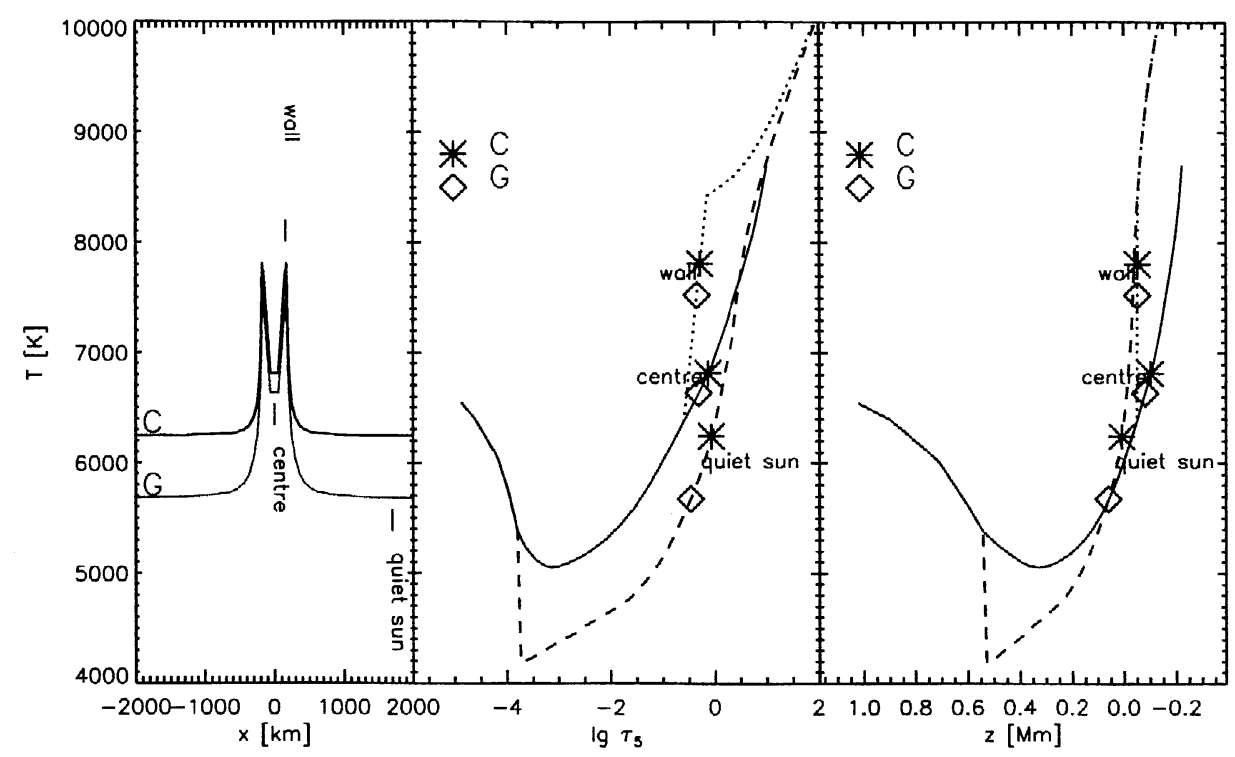

Figure 1. Left panel displays outgoing intensity in the form of $T_{\text {rad }}$. Right panels show temperature along the three lines of sight marked in the first panel. The marks show where $T_{\text {kin }}=T_{\text {rad }}$.

with respect to the quiet sun is greater in $G$ than in $C$ mainly because the photosphere is darker in the $\mathrm{G}$ band.

A detailed inspection of the synthetic spectra shows how most lines get weaker over the flux tube. We interpret this as being caused by the shallower temperature gradient and the lower density inside the flux tube. Molecular lines are particularly sensitive due to the pressure dependence of molecular equilibria. Thus bright points should show up particularly well in molecular lines.

\section{Comparison with observations}

Figure 2 compares the contrast values of the two passbands - i.e. the integrated intensity $(G$ and $C)$ normalised to the quiet sun intensity $(\langle G\rangle$ and $\langle C\rangle)$ with the observations. The points and contours represent pixel intensities in the images. The stars are maximum intensities from the flux tube after convolution with a range of smearing functions simulating the effects of the atmosphere and the telescope. The resulting maximum contrast of the flux tube is very sensitive to the far wings of the smearing profile - which are very difficult to assess. Anyway it seems the results are not in disagreement with the observations. Note again that the observational points are pixel values while the synthetic intensities represent bright-point maxima. 


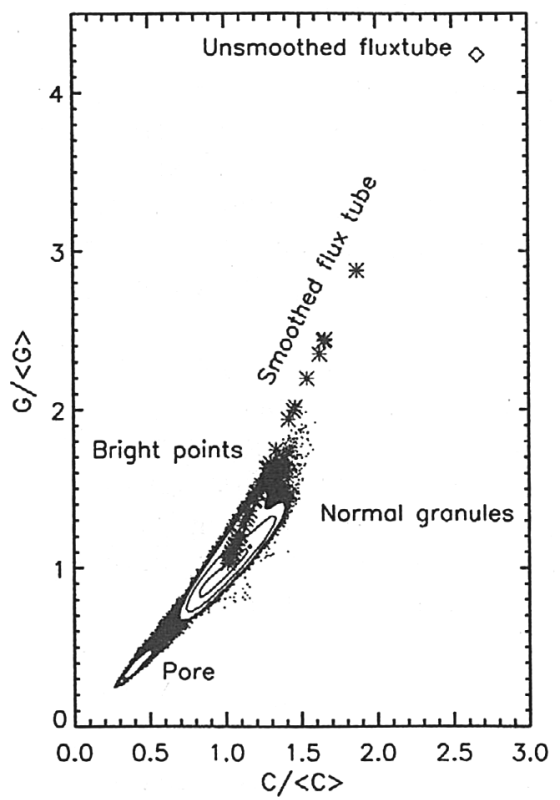

Figure 2. Observed normalised intensities (points and contours) and synthetic results with a range of different smoothing applied (stars and diamond).

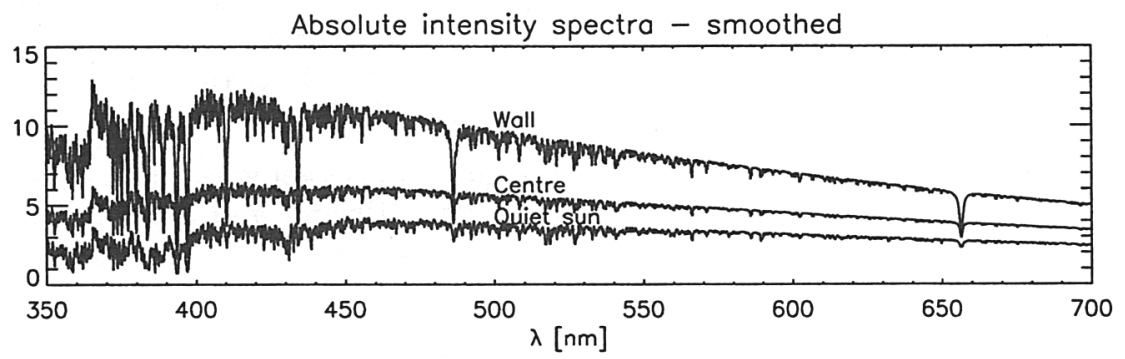

Intensity ratio: Flux tube wall/Quiet sun



Figure 3. The entire optical spectrum from the three lines of sight of the flux-tube model. Below that, the resulting maximum bright-point contrast to the quiet photosphere. 


\section{Flux-tube signatures in other bands?}

Could there be other spectral regions useful for proxy magnetometry or fluxtube diagnostics? We synthesised also a wide range of the solar spectrum with $R=20000$. The upper panel of Fig. 3 shows smoothed spectra from the same three locations of the flux tube, and the wall/quiet-sun contrast. The Balmer lines stand out as they get stronger due to the high temperature of the flux-tube walls. But the Balmer jump does not get particularly strong, so that feature is not predicted to be a good diagnostic.

The violet $\mathrm{CN}$ band gives a strong contrast signal. This may be a candidate band pass and is known to show the photospheric network well (Chapmann 1970). Inspection of the molecular equilibrium shows that $\mathrm{CN}$ is depleted relatively more than $\mathrm{CH}$ in the flux tube. Preliminary results from the Swedish Vacuum Solar Telescope on La Palma received during the symposium (Rouppe van der Voort, private communication) confirm this prediction in that $\mathrm{CN}$ bright points look very similar to those seen in the $\mathrm{G}$ band.

\section{Conclusions}

The Briand \& Solanki model produces bright-point contrasts similar to those observed. But the spectrum synthesis was made under the LTE assumption and the photospheric part of the flux tube was constructed from LTE inversions of spectropolarimetric data with refinements from 1D NLTE spectral modelling. Do we believe in LTE? Not in general. Even 1D NLTE could be looked upon with suspicion in this context. A contrasting scenario would be one where UV radiation from the hot walls photodissociates $\mathrm{CH}$ in the flux tube and causes overionisation of metals, thus affecting the semi-empirical modelling. If there is any place in the solar photosphere where 3DNLTE effects could be important it should be in these flux tubes.

Acknowledgments. Mats Löfdahl and Tom Berger generously shared their observational data before publication. Carine Briand and Sami Solanki very kindly provided their flux-tube models and software to handle them plus instructions.

\section{References}

Berger, T.E., Schrijver, C.J., Shine, R.A., Tarbell, T.D., Title, A.M., Scharmer, G. 1995, ApJ, 454, 531

Chapman, G.A. 1970, Sol. Phys., 13, 78

Briand, C., Solanki, S.K. 1995, A\&A, 299, 596

Bruls, J.H.M.J., Solanki, S.K. 1993, A\&A, 273, 293

Solanki, S.K. 1986, A\&A, 168, 311 